\title{
Oral Dextrose Gel for Neonatal Hypoglycemia and Delayed Newborn Bath's Effects on the In-Hospital Exclusive Breastfeeding Rate
}

\section{Rana Alissa*MD, Patty Williams APRN Sandy Inman IBCLC Mandy Schumacher MSN IBCLC RN, Anuta Ciurte BSN RN IBCLC Carmen Smotherman MS MPH and Amr Matoq MD}

University of Florida College of Medicine Jacksonville

Submission: July 30, 2020 Published: February 04, 2021

*Corresponding author: Rana Alissa, MD, University of Florida College of Medicine Jacksonville, 655 West Eighth Street, Jacksonville, FL 32209 , Tel: 904-244-3508; Fax: 904-244-4301

Abstract

Background: World Health Organization recommends exclusive breastfeeding in the first 6 months of an infant's life. Exclusive breastfeeding is the use of human milk as the only food source. Exclusive breastfeeding at the time of infant's discharge from Mother-Baby unit increases the likelihood of exclusive breastfeeding throughout the neonatal period. A recent study demonstrated that the use of oral dextrose gel as first-line treatment for neonatal hypoglycemia stabilized blood glucose and reduced transferring the neonates to specialized units to receive intravenous dextrose. Additionally, delaying the initial newborn bath has been shown to increase the likelihood of exclusive breastfeeding at hospital discharge due to the lack of interruption in breastfeeding initiation and skin-to-skin care.

Research Aim: To improve in-hospital exclusive breastfeeding rate by adopting the use of oral dextrose gel for neonatal hypoglycemia and by delaying newborn baths to12 hours of life and beyond.

Method: We implemented oral dextrose gel in March 2015 and adopted newborn bath delay to 12 hours of life and beyond in May 2015. Newborns who were exclusively breastfed at discharge from Mother-Baby unit, qualified for the study. We determined the core measure PC-05 (the in-hospital exclusive breastfeeding rate) in the 8 months before and after interventions.

Result: The rate of exclusive breastfeeding increased from $20.7 \%$ in the 8 months before the first intervention to $32.8 \%$ in the 8 months after the second intervention.

Conclusion: Implementation of oral dextrose gel for neonatal hypoglycemia and delay initial newborn bath were associated with improving our in-hospital exclusive breastfeeding rate.

\section{Background}

To achieve optimal growth, development, and health, World Health Organization (WHO) recommends that infants should be exclusively breastfed in the first six months of life [1]. WHO defines exclusive breastfeeding as "the use of only breastmilk for the provision and maintenance of fluid and nutritional needs and the omission of other food or liquids, including water, with the exception of oral rehydration solution, drops, or syrups consisting of vitamins, mineral supplements, or medicines as needed" [2].

The Centers for Disease Control and Prevention's (CDC's) breastfeeding report card, which was published on August 20, 2018, using the National Immunization Surveys (NIS) [3], stated that among infants born in 2015 in the U.S. or its territories, $83.2 \%$ had ever been breastfed; $57.6 \%$ were breastfed some in the first 6 months of life; and only $25 \%$ were exclusively breastfed in the first 6 months of life. This was very close to the $25.5 \%$ rate of exclusive breastfeeding in the first 6 months of life according to Healthy People 2020 goals and objectives [4].

The Baby Friendly Hospital Initiative, also known as the Baby Friendly Initiative, is a worldwide program of United Nations International Children's Emergency Fund (UNICEF) and WHO, which was launched in 1991 and revised in 2018 [5], to help motivate facilities to provide global maternity and newborn 
services and implement the Ten Steps to Successful Breastfeeding. The ten steps summarize a package of policies and procedures that facilities providing maternity and newborn services should implement to support breastfeeding [5]. WHO has recommended all facilities worldwide providing maternity and newborn services to implement the ten steps.

Step 4 (Baby-Friendly USA, 2016) [6] discusses helping mothers initiate breastfeeding within one hour of birth. All infants should be given to their mothers to be held with uninterrupted and continuous skin-to-skin contact immediately after birth and until the completion of the first feeding; a delay in infant bathing until whenever feasible is recommended [6].

In 2007, Petrova et al. [7] stated: "mothers who practice exclusive breastfeeding in-hospital are more likely to exclusively breastfeed throughout the neonatal period" [7]. A review for neonatal hypoglycemia [8] acknowledges that neonatal hypoglycemia is one of the most common causes for transfer to Neonatal Intensive Care Unit (NICU). In 2013, Harris et al. [9] recommended the consideration of oral dextrose gel as first-line management for late preterm and term infants with neonatal hypoglycemia [9]. In 2016, Weston et al. [10] suggested that oral dextrose gel was able to keep infants and mothers together without the need to transfer infants to a higher care unit and improved the rate of exclusive breastfeeding after discharge from the hospital [10].

All these observations stimulated us to review our MotherBaby unit policies and procedures to determine what we could modify to increase our rate of exclusive breastfeeding at the time of discharge. We identified two opportunities for intervention: modification of our management of neonatal hypoglycemia, a relatively common condition especially among certain high-risk newborns, and delay of the initial newborn bath. Such efforts also responded to an expansion in the report of hospital metrics required by the Joint Commission [11].

In December 2012, the Joint Commission mandated that hospitals with large delivery services (defined as those with greater than 1,100 births per year) report on the full perinatal care (PC) core measure set. This set included the following 5 metrics: PC-01: Elective Delivery; PC-02: Caesarean Section; PC-03 Antenatal Steroids; PC-04: Health Care-Associated Bloodstream Infection; and PC-05: Exclusive Breastfeeding [11].

Risk factors for neonatal hypoglycemia include prematurity, diabetic mother, small for gestational age (SGA) and large for gestational age (LGA) status [12]. The American Academy of Pediatrics (AAP), breastfed infants tolerate lower plasma glucose concentrations without any clinical manifestations or sequelae of neonatal hypoglycemia because of the increased ketone concentrations [12]. Neonatal hypoglycemia is not uncommon and can result in significant long-term neurodevelopmental morbidity [12].

AAP clinical guidelines for neonatal hypoglycemia include frequent pre-prandial point of care evaluations of blood glucose levels in high-risk newborns every 2-3 hours with interventions corresponding to the level of blood glucose and the hours of age of the infant [12]. If the blood glucose level is normal, newborns are fed, and the glucose level is rechecked before the next feed. However, the guidelines recommend interventions in infants with subnormal glucose levels ranging from supplemental feedings (usually with formula) to administration of intravenous dextrose, which generally necessitates the infant's transfer to a specialized newborn unit. Such transfers make it exceedingly difficult to achieve exclusive breastfeeding through hospital discharge [10].

Moreover, "early bathing disrupts breastfeeding and skin-toskin care in the first hours of life and is likely to reduce the rate of exclusive breastfeeding at the time of discharge", Preer et al. [13] stated.

\section{Research Aim}

To improve our in-hospital exclusive breastfeeding rate at the time of discharge

\section{Methods}

\section{Design}

To meet the study, aim, we implemented two sequential care interventions in our Mother-Baby unit: oral dextrose gel and delay of newborn bath.

Our first intervention was to implement the use of oral dextrose gel to manage neonatal hypoglycemia for all infants admitted to our Mother-Baby unit, and who are at risk for hypoglycemia: prematurity (35-36/7 weeks gestational age), diabetic mother, SGA and LGA. Therefore, we changed our hospital's neonatal hypoglycemia policy to adopt the use of oral dextrose gel in compliance with the AAP guidelines on the management and treatment of neonatal hypoglycemia [12] (Figures 1 \& 2). We simply added oral dextrose gel to breastfeeding in each situation where the AAP guidelines recommended refeeding due to a hypoglycemic episode. The use of oral dextrose gel was implemented in our hospital's Mother- Baby unit policy regarding the management of neonatal hypoglycemia, which was approved by our hospital's Bylaws Committee to become the official policy and the standard of care for the management of neonatal hypoglycemia for all newborns with neonatal hypoglycemia, and not only for newborns enrolled in this study.

Our second intervention was to delay the bath of all newborn infants admitted to our Mother-Baby unit to a minimum of 12 hours of life unless the parent(s) requested an earlier bath and/or in instances of maternal chorioamnionitis, hepatitis C, hepatitis B and Human Immunodeficiency Virus (HIV), which are indications for immediate bath after birth. We also changed our hospital's Mother-Baby unit policy regarding the delayed bath accordingly to become the standard of care for all newborns admitted to our Mother-Baby unit and not only for newborns enrolled in this study. 


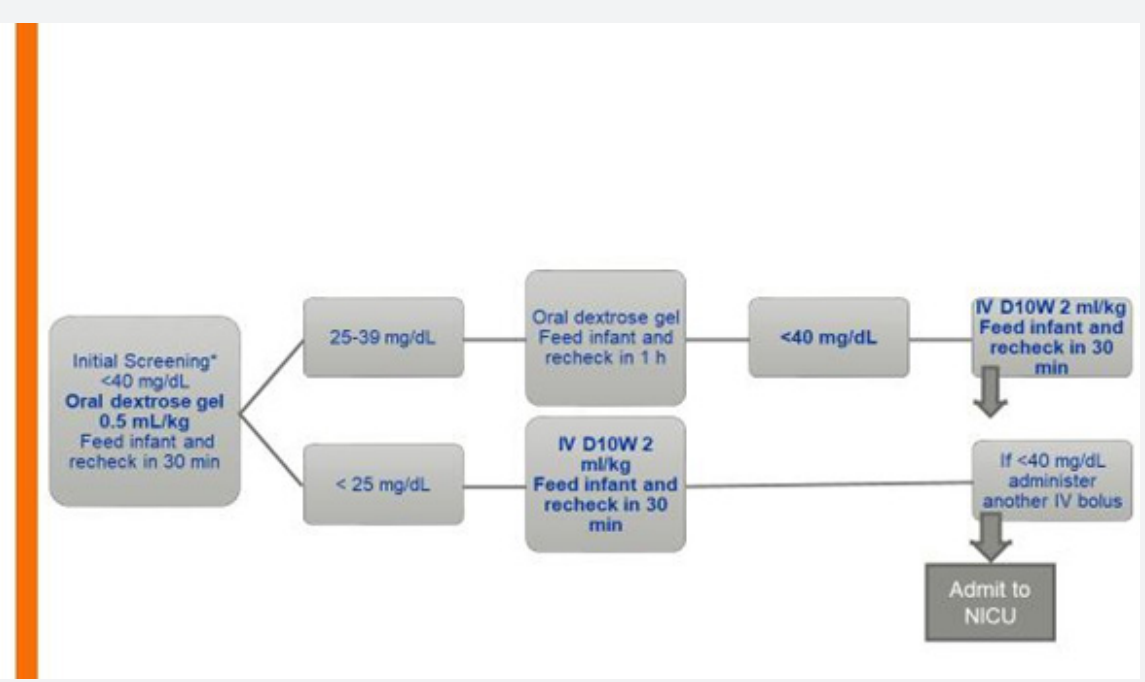

Figure 1: Hypoglycemia protocol (0 to $4 \mathrm{~h})$.

Identify infants at risk (SGA, LGA, Preterm, and IDM)

Feed infant within the first hour

Check glucose 30 min after the first feed.

h: hour, min: minutes, NICU: Neonatal Intensive Care Unit, SGA: Small for Gestational Age, LGA: Large for Gestational Age, IDM: Infant of Diabetic Mother

*If Accucheck screening is low, send confirmatory serum glucose level-If baby is symptomatic and glucose $<40 \mathrm{mg} / \mathrm{dl}$, give IV D10W

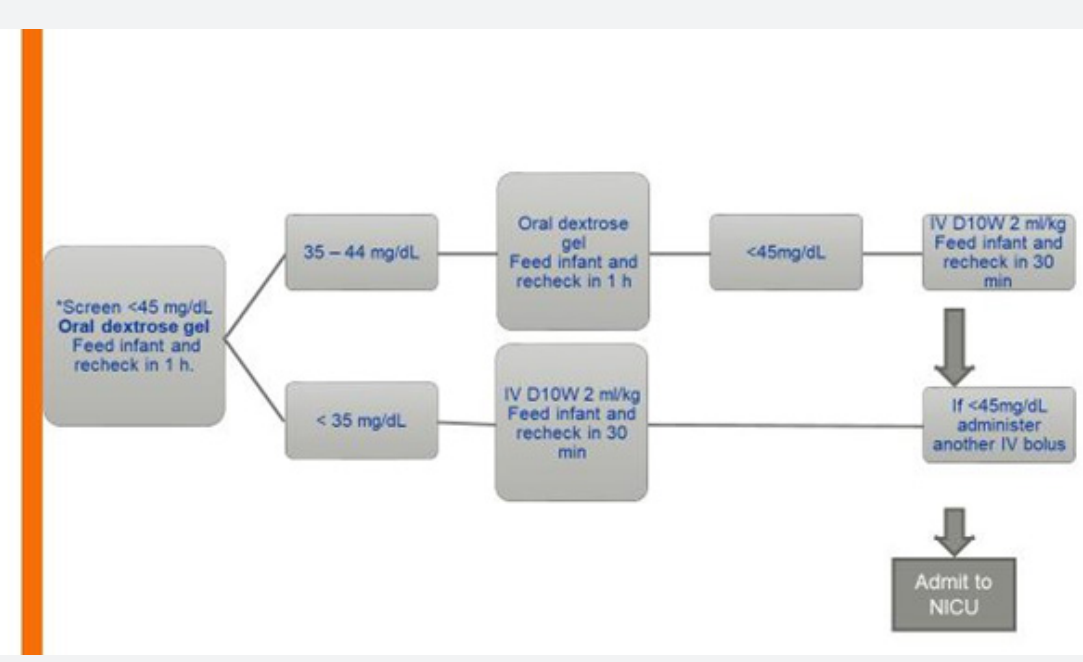

Figure 2: Hypoglycemia protocol $(4-24 \mathrm{~h})$

Screen every $2-3 \mathrm{~h}$, before feeds.

h: hour, min: minutes, NICU: Neonatal Intensive Care Unit, SGA: Small for Gestational Age, LGA: Large for Gestational Age, IDM: Infant of

Diabetic Mother

*If Accucheck screening is low, send confirmatory serum glucose level

- If screening $>45$, continue screening every 2-3 for $12 \mathrm{~h}$ (IDM and LGA) or $24 \mathrm{~h}$ (Preterm and SGA).

- Must have at least 2 consecutive values $>45$ prior to discontinue hypoglycemia protocol

- If the infant is symptomatic and glucose $<40 \mathrm{mg} / \mathrm{dl}$, administer IV D10W

We implemented the use of oral dextrose gel in March 2015 and adopted the delay in the initial newborn bath to 12 hours of life and beyond in May 2015. We determined the Joint Commission core measure PC-05 (i.e., the in-hospital exclusive breastfeeding rate) in the 8 months before and the 8 months after interventions.
Our project was submitted to the Institutional Review Board (IRB) in November 2015 as a Quality Improvement retrospective cohort study. The IRB indicated that no approval is required since no patient information is collected, only the rate of exclusive breastfeeding at the time of discharge. However, per IRB, an official form had to be requested. The submitted form requested 
data on all infants discharged from our Mother-Baby unit who were exclusively breastfed at the time of discharge. The period of requested data was July 2014 to January 2016. The report was generated by the Office of Information Technology, per the study's required parameters, in January 2016.

We defined exclusive breastfeeding as only human milk feeding from the time of birth until the time of discharge to home from the hospital. The staff, including attending physicians, nurse practitioners, residents, nurses, nurse assistants, and lactation consultants, received in-service education prior to implementation of the two interventions with constant emails and meetings for continuous updates and education after implementation of the two interventions.

\section{Setting}

We conducted this project in our hospital that averages 189 deliveries per month. Our Mother-Baby unit is level 1 that admits term and late preterm infants $\geq 35$ weeks gestational age and weigh $\geq 2000$ grams.

Oral dextrose gel was implemented in March 2015, and the delay in the newborn bath was implemented in May 2015. Data were collected on the rates of exclusive breastfeeding at the time of discharge on all infants during two consecutive time periods: Period 1: 8 months before the first intervention (i.e., the implementation of oral dextrose gel) July 2014- February 2015, and Period 2: 8 months after the second intervention (i.e., the delay in the newborn bath for 12 or more hours) June 2015January 2016. The data collected in the period between the two interventions was excluded from the analysis.

At our hospital, newborns are discharged home with their mothers between 24 and 72 hours. Rarely, mothers with medical complications remain admitted for longer period depending on their medical condition. Regardless of the length of stay of the newborn, exclusive breastfeeding is defined at the time of infant's discharge.

\section{Sample}

Inclusion Criteria: all infants admitted to our Mother-Baby unit who were exclusively breastfed at the time of discharge were included in the study. Exclusion Criteria: all infants transferred from the Mother-Baby unit to the NICU for any reason and all infants who received any amount of formula during their inhospital stay.

\section{Measurement}

A segmented regression analysis of interrupted time series was used by the Office of Statistics/ Data Solution Center, where the data were analyzed and graphed to evaluate the level and trend changes in the breastfeeding rate. This method allowed us to assess, in statistical terms, how much the intervention changed the breastfeeding rate immediately and over time [14].

Observations nearer together in time are often more strongly correlated; thus, an interrupted time series had to be estimated using an autoregressive form. This was accomplished in Statistical Analysis System (SAS) using PROC AUTOREG [15]: "The DurbinWatson statistic was used to test for the serial autocorrelation of the error terms in the regression model. Values close to 2.0 indicate no serious autocorrelation".

\section{Data Collection}

Our study team included lactation consultants, pediatric resident, nurse practitioner, statistician and the attending pediatric physician received the data generated by the Office of Information Technology in January 2016. The entire team except for our statistician helped organizing the data then submitted it to our statistician for data analysis.

Obtaining consent from parents to enroll their infants in the study was not required, since management of these infants was the standard of care in our Mother-Baby unit.

\section{Data analysis}

The regression model to estimate the level and trend in the rate of breastfeeding before the intervention and the changes in the level and trend following the intervention was as follow:

"Percent $t=\beta \_0+\beta_{-} 1 *$ time_t $t+\beta \_2 *$ intervention_t $+\beta_{-} 3^{*}$ [time afer intervention) $\mathrm{t} t+\mathrm{e}_{-} \mathrm{t}$ "

Here, Percent $t$ is the rate of breastfeeding in month $t$; time $t$ is a continuous variable indicating the time in months at time $t$ from the start of the observation period (baseline trend); intervention $t$ is an indicator for time t occurring before (intervention $=0$ ) or after (intervention=1) the intervention; and time after intervention $t$ is the number of months after the intervention at time $t$, which is coded 0 before the intervention or sequentially numbered for time periods after implementation. In this model, $\beta_{-} 0$ estimates the baseline breastfeeding rate at time zero; $\beta \_1$ estimates the change in the breastfeeding rate that occurs with each month before the intervention; $\beta \_2$ estimates the level of change in the breastfeeding rate immediately after the intervention; and $\beta_{-} 3$ estimates the monthly change in the breastfeeding rate after the intervention compared with the monthly trend before the intervention. The error term e_t at time $t$ represents the random variability not explained by the model. Stepwise elimination of non-significant terms was used to find the most parsimonious regression model.

\section{Results}

After controlling for autocorrelation, the Durbin-Watson statistic for the regression model was $2.184(\mathrm{P}=0.304$ for the hypothesis of positive autocorrelation and $\mathrm{P}=0.695$ for the hypothesis of negative autocorrelation), which indicated no autocorrelation. The results presented in Table 1 indicate that immediately preceding the beginning of the observation period, the estimated rate of breastfeeding was $17.5 \%$. There was no significant month-to-month change in the breastfeeding rate before the intervention $(\mathrm{P}=0.178)$. Immediately after the intervention, the estimated rate of breastfeeding increased by $8.7 \%(\mathrm{P}=0.021)$. There was no significant change in the month- 
to-month trend in the breastfeeding rate after the intervention $(\mathrm{P}=0.486)$. After the stepwise elimination of non-significant terms, the most parsimonious model contained only the intercept and the significant level of change in the breastfeeding rate (Table 1). We estimated that after the interventions, the monthly breastfeeding rate increased by $12.1 \%$, and had the intervention not been introduced, the breastfeeding rate per month would have been $20.7 \%$. Thus, the breastfeeding rate increased $58.7 \%$ after the intervention to $32.8 \%$.

The average rate of exclusive breastfeeding in the 8 months before the first intervention (period 1) was $20.7 \%$, and the rate of exclusive breastfeeding after the second (period 2) increased to an average of $32.8 \%$ with a peak of $40 \%$ during this period (Figure 3 \& Table 2).

Table 1: Parameter Estimates from the Segmented Regression Model Predicting the Breastfeeding Rate.

\begin{tabular}{|c|c|c|c|c|c|}
\hline Variable & DF & Parameter Estimate & Standard Error & $t$ Value & P-value \\
\hline \multicolumn{6}{|c|}{ a. Full segmented regression model } \\
\hline Intercept & 1 & 0.1746 & 0.0252 & 6.93 & $<.0001$ \\
\hline Time & 1 & 0.0071 & 0.0049 & 1.43 & 0.1783 \\
\hline Intervention & 1 & 0.0872 & 0.0327 & 2.67 & 0.0206 \\
\hline Time after intervention & 1 & -0.005 & 0.0071 & -0.72 & 0.4859 \\
\hline \multicolumn{6}{|c|}{ b. Most parsimonious segmented regression model } \\
\hline Intercept & 1 & 0.2067 & 0.0115 & 17.95 & $<.0001$ \\
\hline Intervention & 1 & 0.1214 & 0.0163 & 7.45 & $<.0001$ \\
\hline
\end{tabular}

DF: Degrees of Freedom

Table 2: Summary Statistics for Exclusive Breastfeeding Rates by Period.

\begin{tabular}{|c|c|c|c|c|c|}
\hline Period & $\begin{array}{l}\text { Period start-end } \\
\text { date }\end{array}$ & $\begin{array}{l}\text { Average } \\
\text { Rate }\end{array}$ & SD & Minimum* & Maximum* \\
\hline Period 1: 8 months before the first intervention & $\begin{array}{c}\text { July } 2014-\text { February } \\
2015\end{array}$ & 20.7 & 2.8 & 17.6 & 26.4 \\
\hline \multicolumn{6}{|c|}{ First Intervention - Implementation of oral dextrose gel in March 2015} \\
\hline \multicolumn{6}{|c|}{ Second Intervention - Implementation of delayed newborn bath in May 2015} \\
\hline Period 2: 8 months after the second intervention & $\begin{array}{c}\text { June } 2015 \text { - January } \\
2016\end{array}$ & 32.8 & 3.7 & 28.0 & 40.0 \\
\hline
\end{tabular}

\section{SD: Standard Deviation.}

*The minimum and maximum are the minimum and maximum monthly rate during the described period.

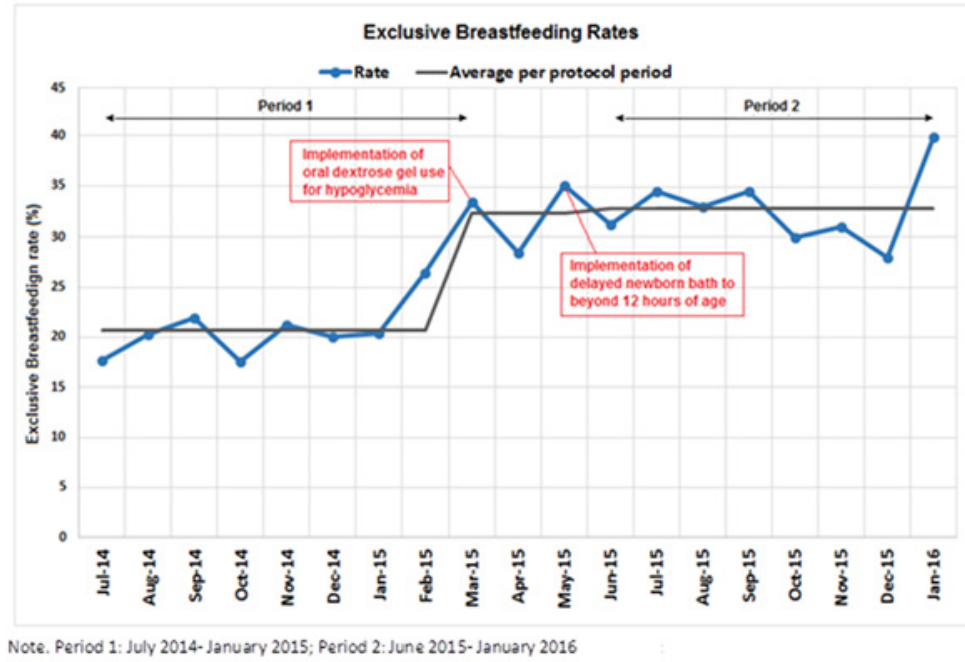

Figure 3: Exclusive breastfeeding rates in period 1 and period 2.

Period 1: July 2014- January 2015; period 2: June 2015- January 2016. 


\section{Discussion}

The AAP Clinical Guidelines on neonatal hypoglycemia [12] state that "Any approach to neonatal hypoglycemia management needs to account for the overall metabolic and physiologic status of the infant and should not unnecessarily disrupt the motherinfant relationship and breastfeeding."

In a review of the literature, we found supportive evidence that our first intervention, oral dextrose gel, is a simple, safe and costeffective method to treat neonatal hypoglycemia [16]. It reduced the need to admit newborns at risk for hypoglycemia to the NICU for intravenous dextrose treatment [17]. An initial report by Harris et al. [9] described the use of the oral dextrose gel compared to placebo reduced the need for intravenous dextrose from $24 \%$ to $14 \%$ (relative risk, $0.57 ; 95 \%$ CI $0.33-0.98, \mathrm{P}=0.04$ ). Weston et al found that infants treated with oral dextrose gel were less likely to be separated from their mothers for treatment of hypoglycemia and more likely to be exclusively breastfed after discharge [10].

The second intervention, delaying the initial newborn bath for 12 hours was also shown to decrease the rate of hypoglycemia by $50 \%$ in high-risk infants in one report [18], and was associated with increased in-hospital exclusive breastfeeding rate along with maternal feeding plan to use human milk after discharge [19].

We implemented both interventions not only to reduce the frequency of NICU admission of newborns with hypoglycemia but also to increase our rate of exclusive breastfeeding. After implementation of these two interventions, we did witness an increase in the rate of exclusive breastfeeding from a mean of $20.7 \%$ to a mean of $32.8 \%$.

This increase in the breastfeeding rate was associated with the two interventions implemented as shown by the data in Table 2; the exclusive breastfeeding rate during period 1 was lower than the exclusive breastfeeding rate during period 2 .

The Mother-Baby staff members were periodically updated, educated, and reminded of the interventions. The importance of the oral dextrose gel and delayed newborn bath in improving the exclusive breastfeeding rate in infants with hypoglycemia was the primary focus of the continued updates and education. The staff members' education, although not a key part in the results of our study, played an important part in our success.

Multi centers with larger number of newborn enrollment is needed to address the benefits of oral dextrose gel and/or delay newborn bath on improving the rate of exclusive breastfeeding at the time of discharge from the hospital and also until 6 months of life to meet the WHO and AAP recommendation on exclusive breastfeeding, given the numerous benefits of human milk.

Hospitals that wish to improve their performance on the Joint Commission PC-05 metric can consider adopting one or both interventions.

\section{Limitations}

Our study was conducted retrospectively at a single center with a relatively small sample size. We used a before-and-after study design as opposed to a design in which one group received an intervention and another group, the control group, did not receive an intervention. Data on infants who had immediate bath because of parental request and/or because of maternal chorioamnionitis, hepatitis C, hepatitis B and HIV, although it is a small sample, it was not collected. Data specific to each intervention was not collected. Finally, the fact that our study was not blinded increased the risk of selection bias.

\section{Conclusion}

In our institution, we increased the exclusive breastfeeding rate at the time of discharge after we implemented oral dextrose gel and delay in the newborn bath.

\section{References}

1. https://www.who.int/news/item/15-01-2011-exclusivebreastfeeding-for-six-months-best-for-babies-everywhere

2. World Health Organization, \& LINKAGES (2009) Infant and Young Child Feeding: Model Chapter for Textbooks for Medical Students and Allied Health Professionals. Geneva: World Health Organization.

3. h t t p s : / / w w w. c d c. g o v / b r e a s t f e e d i n g/ pdf/2018breastfeedingreportcard.pdf

4. http://www.usbreastfeeding.org/p/cm/ld/fid=221

5. https://www.who.int/nutrition/publications/infantfeeding/bfhiimplementation-2018.pdf

6. Baby-Friendly USA (2016) Guidelines and Evaluation Criteria for Facilities Seeking Baby-Friendly Designation. Baby-Friendly USA , Albany, NY.

7. Petrova A, Hegyi T, Mehta R (2007) Maternal race/ethnicity and onemonth exclusive breastfeeding in association with the in-hospital feeding modality. Breastfeed Med 2(2): 92-98.

8. Alsaleem A, Saadeh L, Kamat D (2019) Neonatal Hypoglycemia: A Review. Clin Pediatr (Phila) 58(13): 1381-1386.

9. Harris DL, Weston PJ, Signal M, Chase JG, Harding JE (2013) Dextrose gel for neonatal hypoglycemia (the Sugar Babies Study): A randomized, double-blind, placebo-controlled trial. Lancet 382(9910): 2077-2083.

10. Weston PJ, Harris DL, Battin M, Brown J, Hegarty JE, et al. (2016) Oral dextrose gel for the treatment of hypoglycemia in newborn infants. Cochrane Database of Systemic Reviews 5(5): CD011027.

11. The Joint Commission. (2013) The perinatal care core measure set per joint commission perspectives. The Joint Commission Perspectives 33(11): 12-14.

12. Adamkin DH, Committee on Fetus and Newborn (2011) Postnatal glucose homeostasis in late-preterm and term infants. Pediatrics 127(3): 575-579.

13. Preer G, Pisegna JM, Cook JT, Henri AM, Philipp BL (2013) Delaying the bath and in-hospital breastfeeding rates. Breastfeed Med 8(6): 485490 .

14. Wagner AK, Soumerai SB, Zhang F, Ross-Degnan D (2002) Segmented regression analysis of interrupted time series studies in medication use research. J Clin Pharm Ther 27(4), 299-309. 
15. SAS Institute Inc. (2008) SAS/ETS 14.1 User's Guide: The AUTOREG procedure. SAS Institute, Cary, NC.

16. Romald J, Coda L, Rishi F, Khalil E (2019) Oral Glucose Gel for Neonatal Hypoglycemia-A single hospital study. Pediatrics $144(2$ MeetingAbstract) 673.

17. Rawat M, Chandrasekharan P, Turkovich S, Barclay N, Perry K, et al. (2016) Oral dextrose gel reduces the need for intravenous dextrose therapy in neonatal hypoglycemia. Biomed Hub 1(3): 1-9.
18. McInerney CM, Gupta A (2015) Delaying the first bath decreases the incidence of neonatal hypoglycemia. Journal of Obstetric Gynecologic and Neonatal Nursing 44(S1), S73-S74.

19. DiCioccio H, Ady C, Bena J, Albert N (2019) Initiative to Improve Exclusive Breastfeeding by Delaying the Newborn Bath. J Obstet Gynecol Neonatal Nurs 48(2): 189-196.
This work is licensed under Creative

Commons Attribution 4.0 Licens

DOI: 10.19080/AJPN.2020.10.555832
Your next submission with Juniper Publishers will reach you the below assets

- Quality Editorial service

- Swift Peer Review

- Reprints availability

- E-prints Service

- Manuscript Podcast for convenient understanding

- Global attainment for your research

- Manuscript accessibility in different formats

( Pdf, E-pub, Full Text, Audio)

- Unceasing customer service

Track the below URL for one-step submission

https://juniperpublishers.com/online-submission.php 